\title{
Developing a Microcomputer Based Automated Testing System for use with Psychogeriatric Patients
}

A. C. CARR, Honorary Lecturer, Department of Psychopharmacology; R. T. Woods, Lecturer in Clinical Psychology and B. J. MOORE, Research Worker, Institute of Psychiatry, De Crespigny Park, London SE5

Microcomputers are increasingly being used in psychiatry for a variety of purposes. ${ }^{1}$ When used in computer-patient 'interviews' they have generally proved acceptable-indeed popular-with psychiatric patients. ${ }^{2}$ Automated psychological assessment is one form of computer-patient interview that is attracting much interest. ${ }^{3}$ Some of the early applications were with elderly patients. ${ }^{4.5}$ Kendrick $^{6}$ has expressed doubts regarding this application with the current generation of elderly people, suggesting that their relative unfamiliarity with computers and video games might cause difficulties.

In a pilot study ${ }^{7}$ we showed that automated testing of psychogeriatric patients with a microcomputer based system was feasible. However, we recommended further development of both equipment and programmes to reduce some of the difficulties that were encountered.

One recommendation was that displays need to be large and clear for the elderly. A fifth of our pilot study sample could not read the instructions and items printed on the TV screen. One snag in simply applying automated tests developed for younger patients ${ }^{8.9}$ is the quantity of written instructions and verbal items used. Two approaches to overcoming the problem are possible; one is to use computer-synthesised speech to back up or supplement written material; the other is to increase the clarity of the written material. We have explored the latter approach.

A second recommendation was the need to avoid the use of response buttons, which introduce an excessive cognitive load in translating the response from screen to response pad. This is avoided if the screen itself is the response device, i.e. by using a 'touch-sensitive screen'. However, Ogden et al. ${ }^{10}$ have argued that a touch-sensitive screen would not be appropriate for elderly people. Three reasons are put forward for this view. Firstly, it is unfamiliar, would appear 'magical' and would tend to be seen as frightening and unpredictable. Secondly, effort would be required to reach the screen, which could be potentially tiring. Thirdly, many elderly people suffering from hypermetropia would need to sit further away than arm's length from the screen, and so could not be in a position both to see the screen and respond appropriately.

Finally, we recommended greater use of the computer's ability to present information graphically and to record response times, making full use of the microcomputer's potential. In the pilot study information and orientation items had simply been presented in a multiple-choice format.

In this study there were then three major aims. Firstly, to examine whether reading difficulties could be reduced by larger, clearer written displays. Secondly, to test whether a touch-sensitive screen would have advantages for elderly patients, despite the contra-indications that have been put forward by Ogden et al. Thirdly, to develop programmes using more of the microcomputer's potential.

\section{Experiment 1-Reducing reading dificulties}

Subjects and equipment: Subjects were consenting in-patients and day-patients from a psychogeriatric assessment unit with various diagnoses, some with dementia. The equipment was a Commodore 64 microcomputer with floppy disc storage, and a 20-inch colour monitor to give as large and distinct a display as possible. Subjects were seated within comfortable reaching distance of the screen and simply asked to read aloud the words as they appeared. Trials were self-paced, responses being recorded by the experimenter. Normal reading glasses were worn as appropriate.

Material: Common four-letter words with frequencies from 7 to 316 per 10,000 were selected ${ }^{11}$ and frequencies balanced across trials.

\section{Test (a) Legibility of standard font}

Eleven subjects completed the test, of mean age 78.4 (s.d. 4.8). Four words were displayed across the screen at each trial. In successive trials the characters were upper-case white on black, lower-case ditto, reverse upper-case (black on white) and reverse lower-case, to a total of 12 trials. Characters were the standard manufacturer's font for the $\mathbf{2 5}$ line, $\mathbf{4 0}$ column display on an $\mathbf{8} \times \mathbf{8}$ dot-matrix.

The percentage of words misread was: upper-case $8.3 \%$, lowercase $10.8 \%$, reverse upper-case $8.3 \%$ and reverse lower-case $11.7 \%$.

Word frequency was significantly associated with reading accuracy $(t=3.68, P<0.01)$, and it was also noted that certain letters were frequently misread (e.g. the computer's ' $W$ ' was often read as ' $H$ ').

Patients' opinions and their speed of response were generally in accord with the above findings, that white-on-black was a little more legible than the reverse, and that upper-case letters were slightly easier to read. Nevertheless, reading difficulties were unacceptably common in all presentations.

\section{Test (b) Legibility of special large font}

Sixteen patients were tested. Their mean age was 78.9 years (s.d. 6.3 years). Using the computer's programmable character facility, a set of larger and clearer letters was developed each covering four times the area of the standard character. Particular efforts were made to increase the distinctiveness of difficult letters such as ' $w$ ' and ' $\mathrm{I}$ '.

Words were again displayed four at a time across the screen, using the special large characters and the standard characters in alternate trials. All were in upper-case. After all $\mathbf{4 8}$ words had been displayed, the presentation was repeated displaying each word in the opposite character type to that shown previously. Two versions of the test enabled character type at first presentation to be balanced across subjects. 
All 16 subjects completed the test. The mean percentage of errors was 10.6 (s.d. 8.4) for standard characters and 2.8 (s.d. 3.8) for large ones. This difference was significant $(t=5.14, P<0.001)$. The large character set clearly improved these patients' reading accuracy.

\section{Experimeat 2-Matching to sample test}

Subjects: Fifteen day-patients and in-patients from two psychogeriatric assessment units participated. All subjects had a diagnosis of dementia; the diagnosis was based on the history of the person's difficulties, thorough psychiatric and physical examination, CAT scan and other investigations, and cognitive testing. The mean age was 80.6 years (s.d. 5.4). The patients' mean score on a 20 -item memory and information test (available from the authors) was 6.96 (s.d. 5.9; range 0-19).

Equipment: as used in experiment 1 with the addition of a touchsensitive screen to monitor patients' responses. The type of screen used is operated by the subject's finger breaking a two-dimensiona array of invisible infra-red beams arranged across the screen. The computer identifies the position of the subject's finger $(+5 \mathrm{~mm})$ from which beams are broken.

Test design: A visual task was chosen in order to utilise the graphic capabilities of the microcomputer. Two possible paradigms were considered: the traditional matching to sample, where the subject picks out from a group of alternatives the one design that is the same as the sample displayed, and 'odd man out', where the subject picks the one design from a group that differs from the others. In pilot tests we found that patients found both paradigms acceptable and that there was little difference in their performance on the two tasks. Matching to sample was chosen because it allows a wider range in the number of possible responses (odd man out tasks require at least three items)

Each test item involved presentation of a sample design, a common shape (teapot, truck etc.) made up of blocks of three colours, together with a group of designs with the same outline differing only in the internal arrangement of the coloured blocks. Item difficulty could be varied quantitatively by varying the size of the coloured blocks and by varying the number of blocks that were different between the sample and the incorrect response alternatives. Two, three or four response options were provided per trial. Some of the features of the test that were intended to maintain the patient's interest and motivation are listed in Table I. Before the test patients had between six and nine practice trials, of graded difficulty, aimed

TABLE I

Features of matching-to-sample test designed to enhance motivation and interest

1. Use of different colours and colour combinations

2. Written instructions on screen throughout to reduce memory load

3. Instructions and prompts written word-by-word, teletype style, accompanied by auditory prompts to attract the patient's attention

4. Large character set and large TV monitor used to reduce visual problems

5. Immediate auditory feedback for each response from patient

6. Familiar tunes played by computer between items

7. Subject's name incorporated in instructions on screen

8. In practice trials, animation used to demonstrate principle involved and draw attention to correct response

9. Each outline shape resembled a familiar object at shaping and correcting their responses and familiarising them with the test. Twenty-four items made up the test itself; difficulty level, colour, number of options and position of correct response were all presented in balanced order. Patients were seated within comfortable reaching distance of the screen. The experimenter remained with them throughout, providing general encouragement and some additional prompting where necessary. The patient's responses and response latencies were automatically stored on magnetic disk.

\section{Results}

Twelve patients completed this test, most appearing to find it interesting and enjoyable. One other patient had difficulty reading the instructions (despite the large letters), one was unable to grasp what was involved and one more was too restless to complete the test. These patients would have been equally difficult to test by conventional means, and had very low scores on the memory and information test used (mean 1.3).

After one or two trials where the experimenter had to prompt the patient to touch the targets on the screen all were able to do so without difficulty. No patients objected to this mode of response. The test took between 20 and $\mathbf{4 0}$ minutes to complete.

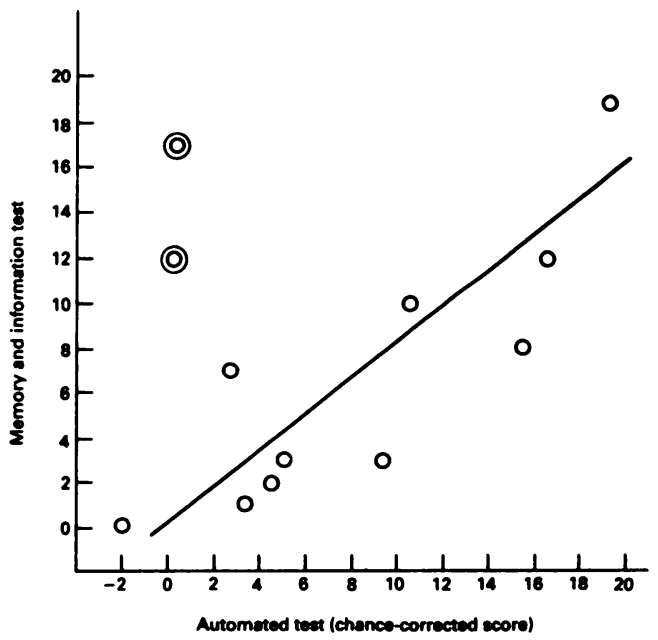

FIG. 1 Scatter-plot of scores of 12 dementing patients on automated matching to sample test against scores on a 20-item memory and information test. Data points for two 'frontal' type patients are circled.

Figure 1 shows a scatter-plot of accuracy on the test against scores on the 20-item memory and information test, which provides an independent measure of degree of dementia. Two subjects stand out from the general trend; review of the case-notes suggested that of the sample these two alone had specific frontal-lobe type difficulties, leading to visuo-spatial problems out of proportion to their level of verbal orientation. Excluding these two subjects, the two measures correlate significantly $(r=0.86, P<0.005)$. For these 10 patients, mean response latencies were also significantly negatively related to the memory and information test score $(r=-0.54, P<0.05)$. Scores on a routinely used behaviour rating scale (available from the authors) covering self-care, ward orientation, dressing and continence 
correlated well both with scores on the automated test $(r=0.79$, $P<0.01)$ and response latencies $(r=-0.83, P<0.01)$.

\section{Comment}

It has proved possible to assess patients suffering from dementia directly at the screen of a microcomputer, using its capabilities to generate graphics and record latencies. A wide range of patients can be tested, from the mildly impaired to those requiring hospital admission. The use of animation, colour and music probably contributed to the enjoyment and interest shown by the majority of patients. The interest aroused is considerable: recently 10 patients have been assessed four times on this test within two weeks, with no signs of reluctance or hesitation. This is of great importance, as few current cognitive tests can be used repeatedly in a short period of time with dementing patients, e.g. during therapeutic trials. The assertion by Kendrick $^{6}$ that the current generation of elderly people would not respond to automated assessment because of their unfamiliarity with TV games fails to find empirical support.

Of course, not all patients are testable by this means-a number are untestable conventionally also. Other types of tests may be needed for those with gross visual deficits and other types of response device for those with gross physical handicaps. Severe restlessness and agitation prevents some patients from sitting down for even a few minutes. Our experience has been, nevertheless, that the majority of psychogeriatric assessment unit patients suffering from dementia could be assessed on this test, including a number thought to require long-term hospital care.

We envisage automated assessment supplementing assessment by clinical psychologists. Conventional testing is a time-consuming procedure, and there are relatively few psychologists who work with the elderly: their assessment should already only be made in special circumstances. ${ }^{12}$ Automated testing will make standardised, structured tests of cognitive function more widely available and allow exact measures of response latency etc. that could not readily be carried out by conventional means.

The improvement in reading accuracy obtained by using large, clear letters is of particular importance in assessing elderly dementing patients. We were surprised at how little help the context gave to these patients when trying to read written instructions. For example, several patients read 'Hatch this' instead of 'Watch this' over a number of trials, despite the confusion such an interpretation caused! The reduction of reading errors will also be of great importance in verbal tests, where words may be presented out of context.

The results from our two 'frontal'-type dementing patients emphasise the limitations of simply assessing memory and orientation alone. However, although there are many relatively well-developed brief orientation tests, there are few comparable tests of other cognitive function. If sub-groups of dementing patients are to be identified ${ }^{13.14}$ the development of such tests is essential.
The use of a touch-sensitive screen as response device has been shown feasible, and fears that it would appear frightening, unpredictable or magical ${ }^{10}$ have not been substantiated. Elderly patients sitting near enough to touch the screen were able to read it without discomfort (with spectacles where appropriate). We have yet to prove that this response device is superior to conventional buttons, and if so whether the difference is enough to justify the considerably greater complexity and expense of a touch-sensitive screen, and perhaps lower reliability.

In conclusion, we have demonstrated that some of the problems encountered previously in automated cognitive assessment of the elderly can be overcome. Reading errors can be greatly reduced with a large, clear character set. A test with a readily understandable paradigm, widely used in animal work, can be presented in an interesting, acceptable manner and provide results for which there is some evidence of validity. The use of a touch-sensitive screen may facilitate the assessment of dementing patients. Finally, different patterns of mental impairment have already begun to emerge from comparison of results of our new test with those from conventional assessment. It is anticipated that the software for this and other automated tests will be available from October 1986. Please contact the authors for details.

\section{ACKNOWLEDGEMENTS}

The authors wish to thank the Medical Research Council, for financial support of this project, and Kim Anthony who assisted in the development of the matching to sample test.

\section{REFERENCES}

'CARR, A. C. \& ANClLL, R. J. (1983) Computers in psychiatry. Acta Psychiatrica Scandinavica, 67, 137-143.

2_- Ghosh, A. \& ANCiLl, R. J. (1983) Can a computer take a psychiatric history? Psychological Medicine, 13, 151-158.

${ }^{3}$ ThOMPson, J. A. \& WILson, S. L. (1982) Automated psychological testing. International Journal of Man-Machine Studies, 17, 279-289.

4GeDYE, J. L. \& Miller, E. (1970) Developments in automated testing systems. In The Psychological Assessment of Mental and Physical Handicap (ed. P. Mittler). London: Methuen, pp. 735-760.

${ }^{5}$ Levy, R. \& Post, F. (1975) The use of an interactive computer terminal in the assessment of cognitive function in elderly psychiatric patients. Age \& Ageing, 4, 110-115.

${ }^{6}$ Kendrick, D. C. (1982) Why assess the aged? A clinical psychologist's view. British Journal of Clinical Psychology, 21, 47-54.

${ }^{7}$ Carr, A. C., Wilson, S. L., Ghosh, A., AnClll, R. J. \& Woods, R. T. (1982) Automated testing of geriatric patients using a microcomputer based system. International Journal of ManMachine Studies, 17, 297-300.

${ }^{8}$ ACKER, W. (1980) In support of microcomputer based automated testing: a description of the Maudsley Automated Psychological Screening Tests (MAPS). British Journal of Alcohol and Alcoholism, 15, 144-147.

'Beaumont, J. G. (1981) Microcomputer aided assessment using standard psychometric procedures. Behaviour Research Methods \& Instrumentation, 13, 430-433. 
${ }^{10}$ Ogden, M., Kellett, J. M., Merryfield, P. \& Millard, P. H. (1984) Practical aspects of automated testing of the elderly. Bulletin of British Psychological Society, 37, 148-149.

"BERGER, K. (1967) The most common words used in conversations. Journal of Communication Disorders, 1, 201-214.

${ }^{12}$ Woods, R. T. \& Britron, P. G. (1985) Clinical Psychology with the Elderly. London: Croom Helm.

${ }^{13}$ MCDONALD, C. (1969) Clinical heterogeneity in senile dementia. British Journal of Psychiatry, 115, 267-271.

${ }^{14}$ Naguib, M. \& LeVY, R. (1982) Prediction of outcome in senile dementia-a computed tomography study. British Journal of Psychiatry 140, 263-267.

\section{Psychogeriatric Liaison A Service to a District General Hospital}

R. M. Fraser, Consultant Psychogeriatrician, and Rosemary Healy, Registrar in Psychiatry, University College

Hospital, London

Liaison psychiatry has been an influential element in hospital psychiatric practice for over a decade now. It is concerned with the 'diagnosis, treatment, study, and prevention of psychiatric disorders among patients in nonpsychiatric health care institutions, especially in general hospitals'. ${ }^{1}$ This paper describes and evaluates a project in which the principles of liaison psychiatry were incorporated into a psychogeriatric service.

South Camden has an elderly (over 65 ) population of 15,000 , an unusually high proportion of whom (43\%) live alone. In October 1979 the consultant psychiatrists responsible for South Camden wrote to their colleagues at University College Hospital (the DGH) regretting "the vexed and growing problem of the waiting list for long-stay psychogeriatric patients". We wrote that, while we would do our best to transfer patients as speedily as possible, it seemed that "many teaching hospital wards are going to have a small number of psychogeriatric patients in residence for the foreseeable future". At that time there was a list of 33 South Camden patients awaiting admission to psychogeriatric wards, and a complicated system of priority ratings within which patients competed for beds as they became available. Eleven of these patients occupied acute hospital beds; the average waiting-time for transfer was 11.5 months.

A psychogeriatric service had been established at the beginning of 1979, and over the course of the next four years the waiting list of patients in the community was reduced until, at the time our study began in October 1983, there were no patients awaiting admission to hospital. However, the problem of 'bed-blocking' in the DGH, though it had become smaller, remained. There were never fewer than four patients awaiting transfer; at the beginning of the study, the average waiting-time was two months.

In 1983 the opportunity arose to assign an additional psychiatric trainee to psychogeriatrics for the equivalent of two sessions weekly; her primary task was that of liaison with the general wards, and it was our main hypothesis that this additional element in the service would bring about, among other benefits, a further substantial reduction in bed-blocking.

\section{Present provisions}

Medical

(i) In-patients There is a 10-bed joint geriatric/psychogeriatric unit at St Pancras Hospital (two miles from UCH) and a 27-bed acute ward at Friern Hospital (eight miles from UCH). These wards are shared equally with South Islington (elderly population 13,000). At Friern Hospital there is a 24-bed female ward and a 23-bed male ward, both for the severely demented; the male ward also receives some patients from North Camden.

(ii) Out-patients There is a twice-weekly clinic at UCH.

(iii) Day-patients There are no day facilities specifically for the elderly mentally ill, but there is a 36-place geriatric day hospital and a 30-place psychiatric day hospital on the St Pancras site, both of whom accept psychogeriatric patients if their needs warrant it. (iv) Junior staffing There is a full time $\mathrm{SHO}$ and a half-time clinical assistant based at Friern. Junior staff on the St Pancras unit are trainee physicians/geriatricians. There had been no UCH psychiatric trainee assigned to the elderly previous to this study.

Local authority

(i) Residential care There are 590 Part 3 places for Camden residents (North Camden elderly population 16,900, South Camden $15,000)$. There are no places specifically for the elderly confused. There are 25 sheltered housing units.

(ii) Day care There are two establishments specifically for the elderly. One is for a mixed group ( 100 places) and the other is for the elderly confused (45 places).

\section{Other}

A community psychiatric nurse for the elderly was appointed in 1983. At the time of the study there was no social worker for the elderly, although one has since been appointed; contact was with the ward-based social worker or with the area team. There is also direct 